\title{
In Situ Analysis of the Membrane-binding Region of Multiple Mitochondrial DNA Molecules in the Mitochondrial Nucleus of Physarum polycephalum
}

\author{
Narie Sasaki ${ }^{1, *}$, Takeshi Suzuki $^{2}$ and Shigeyuki Kawano ${ }^{1}$ \\ ${ }^{1}$ Department of Biological Sciences, Graduate School of Science, \\ University of Tokyo, Hongo 7-3-1, Tokyo 113, Japan \\ ${ }^{2}$ Department of Cell Biology, Institute for Molecular and Cellular Regulation, \\ Gunma University, Showa-machi 3-39-15, Maebashi, Gunma 371, Japan
}

Accepted July 31, 1997

A single mitochondrion contains multiple mitochondrial DNA (mtDNA) molecules in vertebrates (review for Clayton 1991), yeast (Stevens 1977), tobacco (Satoh et al. 1993) and slime mold (Kawano et al. 1983, Sasaki et al. 1994). These multiple mtDNA molecules are organized with proteins to form mitochondrial nuclei (mt-nuclei, review for Kuroiwa 1982) in which replication and segregation of multiple mtDNA molecules are orchestrated. By analogy with the bacterial chromosome (Jacob et al. 1963), it has been assumed that the association of mtDNA with the mitochondrial membrane plays an important role in the mtDNA replication and the segregation of daughter mtDNA molecules (Kuroiwa et al. 1994). The association of mtDNA with the mitochondrial membrane in intact cells has been observed in thin sections with electron microscopy (Nass et al. 1965, Kuroiwa et al. 1977). Thin sections, however, fail to clearly show the association of multiple mtDNA molecules in the mt-nucleus with the membrane in a whole mitochondrion.

The mitochondrion in the slime mold Physarum polycephalum has a rod-like mt-nucleus which contains 18-32 copies of mtDNA (Kawano et al. 1983, Sasaki et al. 1994). The mitochondrial membrane-DNA complex of $P$. polycephalum was isolated and restriction endonuclease analysis of it identified a specific region of mtDNA involved in interaction with the mitochondrial membrane (Kawano and Kuroiwa 1985, Kuroiwa et al. 1994). This membrane-binding region (MBR) was mapped to a $19.6-\mathrm{kbp}$ tandem duplication located on both ends of the 86-kbp linear mtDNA molecule (Takano et al. 1990, Kuroiwa et al. 1994).

To clarify the organization of these multiple MBRs in the mt-nucleus, we localized the sites of the MBRs in the mt-nucleus by fluorescence in situ hybridization (FISH) with a DNA probe specific for the MBR. We found that the MBRs were clustered at a few discrete sites along the mt-nucleus and each cluster contained the MBRs of $10 \mathrm{mtDNA}$ molecules.

Materials and methods

Cultures of cells

An axenic culture of amoebae cell of $P$. polycephalum, strain LU352, was a kind gift from Dr. J. Dee. The amoebae cells were cultured with shaking in axenic medium at $22^{\circ} \mathrm{C}$ as described by Dee (1986).

Microplasmodia of $P$. polycephalum, Colonia isogenic strain KM $182 \times \mathrm{KM} 187$, were cultured in liquid medium at $23^{\circ} \mathrm{C}$ as described by Daniel and Baldwin (1964).

* Corresponding author: Tel: +81-(3)-3812-2111 (ex. 4471); Fax: +81-(3)-3814-1408; E-mail: narie@biol.s.u-tokyo.ac.jp 


\section{Observation of cells}

Cells were fixed on a glass slide in $0.6 \%$ glutaraldehyde and stained with $1 \mu \mathrm{g} / \mathrm{ml} 4^{\prime}$,6-diamidino-2-phenylindole (DAPI) in S buffer $(0.25 \mathrm{M}$ sucrose, $10 \mathrm{mM}$ Tris- $\mathrm{HCl}, \mathrm{pH} 7.7,1 \mathrm{mM}$ EDTA, $\mathrm{pH}$ 7.5, $7 \mathrm{mM}$ 2-mercaptoethanol, $0.75 \mathrm{mM}$ spermidine and 0.4 mM PMSF) and observed under an epifluorescence microscope equipped with a phase-contrast objective (BHS-RFC; Olympus Optical Co., Tokyo, Japan).

\section{In situ hybridization and signal detection}

Cells were washed with distilled water three times and fixed with Carnoy's solution $(25 \%$ glacial acetic acid in ethanol) for $10 \mathrm{~min}$. A drop of fixed cells was then spread on a coverslip, and the coverslip was passed rapidly through a flame to burst and fix the samples. In situ hybridization was performed by the method of Hizume et al. (1992). A 4.7-kbp XbaI/SphI fragment that overlaps a 1.7-kbp region of the MBR of $P$. polycephalum mtDNA (Takano 1990) was used as a probe. DNA was labeled with digoxigenin (DIG)-labeled deoxyuridine-triphosphate using a random primer DNA labeling system (Boehringer Mannheim Biochemicals, Mannheim, Germany). The sample was heat-denatured at $70^{\circ} \mathrm{C}$ for $5 \mathrm{~min}$ and then hybridized with the DIG-labeled DNA probes at $37^{\circ} \mathrm{C}$ for $12 \mathrm{~h}$. After hybridization, the sample was washed with $50 \%$ formamide in $2 \times \mathrm{SSC}(0.3 \mathrm{M}$ sodium chloride, $0.03 \mathrm{M}$ sodium citrate) for $10 \mathrm{~min}$ at $37^{\circ} \mathrm{C}$ and with $2 \times \mathrm{SSC}$ for $10 \mathrm{~min}$ twice. The sample was washed three times in PBS $\left(0.13 \mathrm{M} \mathrm{NaCl}, 7 \mathrm{mM} \mathrm{Na}_{2} \mathrm{HPO}_{4}, 3 \mathrm{mM} \mathrm{NaH} \mathrm{PO}_{4}, \mathrm{pH} 7.2\right)$ and blocked with $15 \mu \mathrm{l}$ of blocking solution (PBS containing 5\% BSA and $0.02 \%$ Tween 20 ) at $25^{\circ} \mathrm{C}$ for $30 \mathrm{~min}$. Then the sample was incubated at $37^{\circ} \mathrm{C}$ for $1 \mathrm{~h}$ with primary anti-digoxigenin mouse monoclonal antibody (Boehringer Mannheim Biochemicals, Mannheim, Germany), which was diluted to $1: 40$ in blocking solution. After washing and blocking again, the sample was incubated at $37^{\circ} \mathrm{C}$ for $30 \mathrm{~min}$ with secondary anti-mouse-immunoglobulin goat antibody conjugated with fluorescein isothiocyanate (FITC, Tago Inc., CA, USA) which was diluted to $1: 40$ in blocking solution. The sample was then washed in PBS, stained with DAPI and observed with an epifluorescence microscope as described above.

\section{Estimation of the amount of miDNA and the length of the mt-nucleus}

Cells were stained with DAPI as described above. The fluorescence intensity emitted from the mt-nuclei was measured with a video-intensified microscope photon-counting system (VIMPCS; Hamamatsu Photonics Ltd. Hamamatsu, Japan) connected to an epifluorescence microscope, as described previously (Kuroiwa et al. 1986). To evaluate the fluorescence intensity of mt-nuclei, T4 phage was used as a DNA standard marker $(1 \mathrm{~T}=170 \mathrm{kbp}$ : Freifelder 1970). Since the $P$. polycephalum mtDNA is $86 \mathrm{kbp}$ in size (Takano et al. 1990), 1T corresponds to about 2 copies of mtDNA. At the same time, the length of each mt-nucleus was measured on the video frame of VIMPCS.

\section{Results and discussion}

The life cycle of $P$. polycephalum is represented by two distinct forms; the haploid uninucleate amoebae and the diploid syncytial plasmodium (reviewed by Goodman 1980). Figures $1 \mathrm{a}$ and $1 \mathrm{~b}$ show DAPI-stained DNA fluorescence images of the amoeba and the plasmodium, respectively. In both the amoeba and the plasmodium, the rod-shaped mt-nuclei were observed around the cell nuclei. The mt-nucleus of the amoeba and the plasmodium contains about 18-24 and $32 \mathrm{mtDNA}$ molecules, respectively, at the logarithmic phase of growth (Kawano et al. 1983, Sasaki et al. 1994).

We used FISH with a DIG-labeled DNA probe specific for the MBR to visualize the sites of the MBRs in the mt-nucleus of amoebae and plasmodia (Figs. 1c-n). When the mt-nuclei were stained with DAPI after FISH, uneven fluorescence intensities were seen in the mt-nuclei (Figs. 1c- 

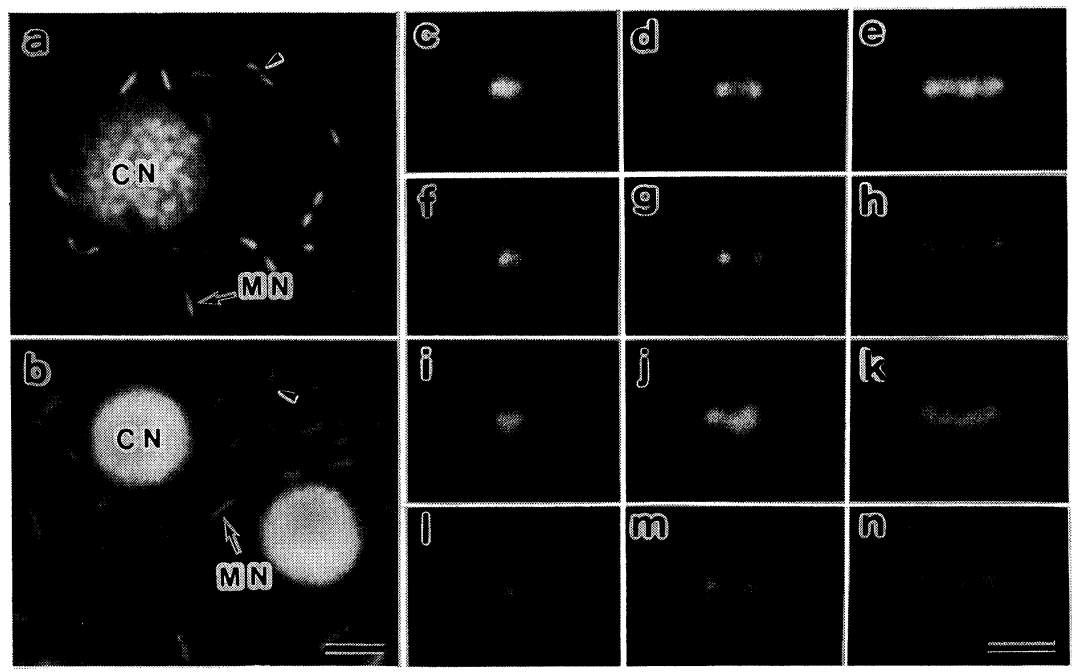

Fig. 1. (a, b) DAPI-stained DNA fluorescence image of amoebae (a) and the plasmodium (b) of $P$. polycephalum. The arrowheads indicate a dividing mt-nucleus. $\mathrm{CN}$, cell nucleus; $\mathrm{MN}$, mt-nucleus. Bar, $3 \mu \mathrm{m}$. $(\mathbf{c}-\mathbf{n})$ The localization of MBRs in the mt-nucleus of amoebae $(\mathrm{c}-\mathrm{h})$ and plasmodium $(\mathrm{i}-\mathrm{n})$. The sites of MBRs in the mt-nucleus were visualized by the FISH with DIG-labeled DNA probe specific for MBR. Sites of MBRs in mt-nuclei ( $\mathrm{f}-\mathrm{h}$ and $\mathrm{l}-\mathrm{n}$ ) are shown together with DAPI-stained DNA fluorescence images (c-e and $\mathrm{i}-\mathrm{k}$ ). The sites of MBRs were detected as a few globular fluorescence along the mt-nucleus. The number of fluorescence in a mt-nuclei increased with an increase in the length of the mt-nucleus.

Bar, $1 \mu \mathrm{m}$.

e, i-k), which probably reflected sites at which DNA probes had hybridized to denatured mtDNA because DAPI stains double stranded DNA. Fluorescence intensities were uniform after FISH without DNA probes (data not shown). In spite of high copy numbers of mtDNA in the mt-nucleus, the sites at which MBR probes had hybridized were observed as a few fluorescence globules distributed along the mt-nuclei (Figs. 1f $-\mathrm{h}, 1-\mathrm{n}$ ). This indicates that MBRs of mtDNA are not distributed randomly throughout the mt-nucleus but are clustered at a few discrete sites along the mt-nucleus.

The number of sites of fluorescence in a mt-nucleus increased with an increase in the length of the mt-nucleus (Figs. 1c-n). Since the length of the mt-nucleus increases as the amount of mtDNA in the mt-nucleus increases (Sasaki et al. 1994), the amount of mtDNA in the mt-nucleus can be estimated from the length. Therefore, we measured the length of the mt-nucleus and quantified the amount of mtDNA in $3600 \mathrm{mt}$-nuclei as described in Materials and methods. It was determined that a mt-nucleus of one- $\mu \mathrm{m}$ in length contained about $12 \mathrm{mtDNA}$ molecules. Fixation with Carnoy's solution for FISH, however, decreased 0.6 time the length of the mt-nuclei such that one- $\mu \mathrm{m}$ mt-nucleus contained about $20 \mathrm{mtDNA}$ molecules. In order to quantify the number of MBRs within a MBR cluster in both amoebae and plasmodia, we investigated the relationship between the number of MBR clusters and the copy number of mtDNA per mt-nucleus (Fig. 2). In both amobae and plasmodia, the number of MBR clusters per mt-nucleus was proportional to the amount of mtDNA per mt-nucleus and one MBR cluster consisted of the MBRs of about 10 mtDNA molecules.

Figure 3 is a model of the organization of multiple mtDNA molecules in a mt-nucleus of $P$. polycephalum. The MBR clusters consisting of MBRs of $10 \mathrm{mtDNA}$ molecules are distributed at a few discrete sites along the mt-nuclei. While the 3.5-kbp MBRs are located in the 19.6-kbp tandem duplication region on opposite ends of mtDNA molecule (Takano et al. 1990, Kuroiwa et al. 1994). Two MBRs of a single mtDNA probably lie adjacent to each other at an MBR cluster. Electron microscopy of serial sections of intact cells indicated that some mtDNA molecules were apparently attached to the mitochondrial inner membrane (Nass et al. 1965, Kuroiwa et al. 1977). Therefore, the 


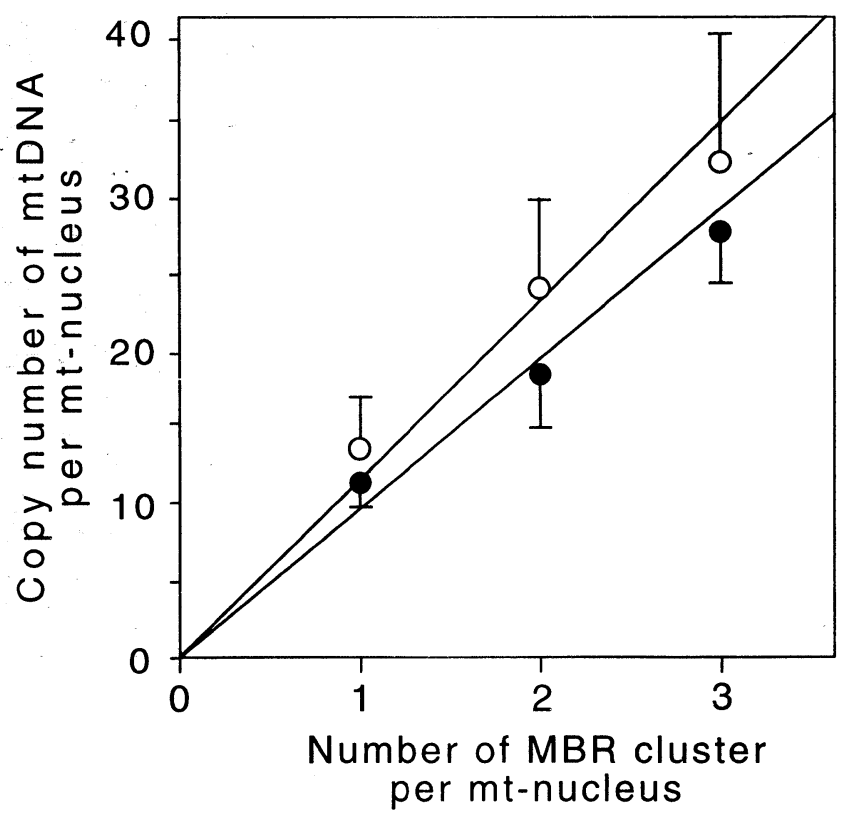

Fig. 2. Relationship between the number of MBR cluster and the copy number of mtDNA in the mtnuclei of amoebae $(O)$ and plasmodia $(\bullet)$, respectively. The copy number of mtDNA per mt-nucleus was calculated from the length of the mt-nucleus, which is exactly proportional to its DNA content. Values are presented as the mean \pm s.d.

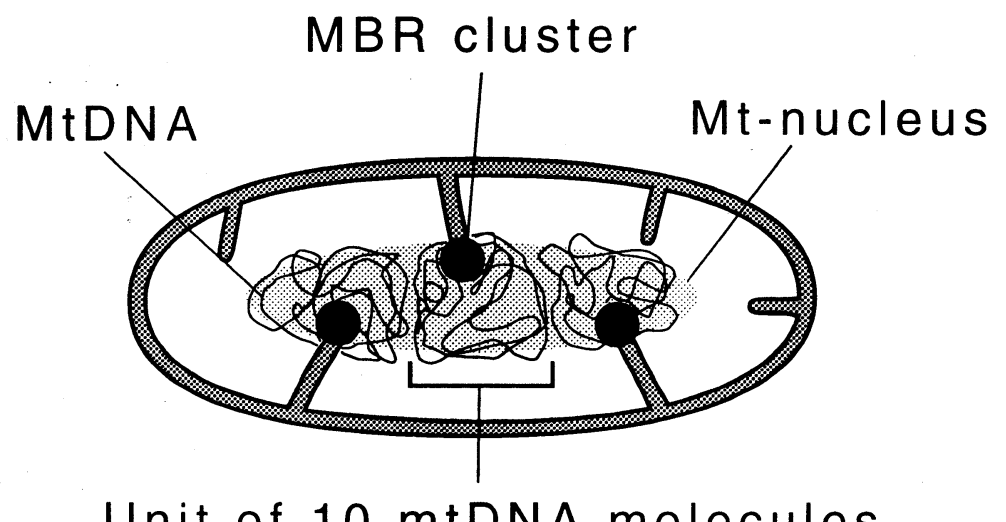

Unit of $10 \mathrm{mtDNA}$ molecules

Fig. 3. A model for the organization of multiple mtDNA molecules in the mt-nucleus. The mitochondrion of $P$. polycephalum has a mt-nucleus containing 18-32 mtDNA molecules. There are a few units consisting of adjacent 10 mtDNA molecules in the mt-nucleus and each unit associates with the membrane at one site of inner membrane.

MBRs of $10 \mathrm{mtDNA}$ molecules may be clustered at one site of inner membrane. The multiple mtDNA molecules in the mt-nucleus should be organized into a few units, each of which is composed of 10 mtDNA molecules, with one MBR cluster. This organization of mtDNA in the mt-nucleus may not change throughout the life cycle of $P$. polycephalum.

The replication of multiple mtDNA molecules in the mt-nucleus is regulated separately in groups of $10 \mathrm{mtDNA}$ molecules termed mitochondrial replicon clusters in both amoebae (Sasaki et al. 1994) and plasmodia (unpublished data). Since the copy number in the unit of mtDNA molecules with one MBR cluster is similar to that in the mitochondrial replicon cluster, these may repre- 
sent the same mt-nuclear structure. This suggests that the organization of mtDNA in the mt-nucleus described here may be important for the regulation of mtDNA replication.

The MBR appears to play an important role in the segregation of mtDNA because the association of mtDNA with the membrane is important for the regulation of mt-nuclear division (Kawano and Kuroiwa 1985) and the MBR is very rich in A-T base pairs, like the centromere which functions in the segregation of chromosomes in the cell nuclei (Alberts et al. 1989). Therefore, the organization of MBRs described here may be important for the regulation of segregation of multiple mtDNA molecules in the mt-nucleus.

\section{Summary}

MtDNA of Physarum polycephalum has duplicated specific regions that bind tightly to the mitochondrial membrane. In this study, we visualized the localization of the membrane-binding regions (MBRs) of mtDNA in the mitochondrial nucleus (mt-nucleus) by fluorescence in situ hybridization with a DNA probe specific for the MBR. The mt-nucleus of $P$. polycephalum contains 18-32 mtDNA molecules. In spite of the high copy number of mtDNA, the sites of MBRs were detected as a few globular fluorescence that were distributed along the mt-nucleus. This indicates that MBRs were clustered at a few discrete sites along the mt-nucleus. Furthermore, the number of MBR cluster in a mt-nucleus was proportional to the number of copies of mtDNA; one MBR cluster consisted of the MBRs of about 10 mtDNA molecules.

\section{Acknowledgments}

This work was supported by a research fellowship to N. S. (no. 3751) from the Japan Society for the Promotion of Science for Young Scientists, and it was also the supported by a Grant-in-Aid for specially promoted Research (no. 06101002) to T. K. from the Japanese Ministry of Education, Science and Culture. We gratefully acknowledge Dr. Jennifer Dee for the kind gift of amoebae cells, strain LU 352, and Dr. Hiroyoshi Takano for supplying the clone of the 4.7-kbp XbaI/SphI fragment.

\section{References}

Alberts, B., Bray, D., Lewis, J., Raff, M., Roberts, K. and Watson, J. D. (e.d.) 1989. In Molecular Biology of the Cell. Garland Publishing, Inc., New York, London.

Clayton, D. A. 1991. Replication and transcription of vertebrate mitochondrial DNA. Ann. Rev. Cell Biol. 7: 453-478.

Daniel, J. W. and Baldwin, H. H. 1964. Methods of culture for plasmodial myxomycetes. In Methods in Cell Physiology. Academic Press, New York.

Dee, J. 1986. The culture of Physarum amoebae in axenic media. In The molecular biology of Physarum polycephalum. Plenum press, New York, 253-269.

Goodman, E. M. 1980. Physarum polycephalum: A review of a model system using a structure-function approach. Int. Rev. Cytol. 63: 1-58.

Hizume, M., Isida, F. and Kondo, K. 1992. Differential staining and in situ hybridization of nuclear organizers and centromeres in Cycas revoluta chromosomes. Jpn. J. Genet. 67: 381-387.

Kawano, S., Nishibayashi, S., Shiraishi, N., Miyahara, M. and Kuroiwa, T. 1983. Variance of ploidy in mitochondrial nucleus during spherulation in Physarum polycephalum. Exp. Cell Res. 149: 359-373.

- and Kuroiwa, T. 1985. Isolation and characterization of a membrane-DNA complex in the mitochondria of Physarum polycephalum. Exp. Cell Res. 161: 460-472.

Kuroiwa, T., Kawano, S. and Hizume, M. 1977. Studies on mitochondrial structure and function in Physarum polycephalum. V. Behavior of mitochondrial nucleoids throughout mitochondrial division cycle. J. Cell Biol. 72: 687-694.

- 1982. Mitochondrial nuclei. Int. Rev. Cytol. 75: 1-59.

—, Miyamura, S., Kawano, S., Hizume, M., Tho-e, A., Miyakawa, I. and Sando, N. 1986. Cytological characterization of 
NOR in the bivalent of Saccharomyces cerevisiae. Exp. Cell Res. 165: 199-206.

-, Ohta, T., Kuroiwa, H. and Sigeyuki, K. 1994. Molecular and cellular mechanisms of mitochondrial nuclear division and mitochondriokinesis. Microscopy Res. Technique 27: 220-232.

Nass, M. N. K., Nass, S. and Afzelius, B. A. 1965. The general occurrence of mitochondrial DNA. Exp. Cell Res. 37: 516539.

Sasaki, N., Suzuki, T., Ohta, T., Kawano, S. and Kuroiwa, T. 1994. Behavior of mitochondria and their nuclei during cell proliferation in Physarum polycephalum. Protoplasma 182: 115-125.

Satoh, M., Nemoto, Y., Kawano, S., Nagata, T., Hirokawa, H. and Kuroiwa, T. 1993. Organization of heterogeneous mitochondrial DNA molecules in mitochondrial nuclei of cultured tobacco cells. Protoplasma 175: 112-120.

Stevens, B. J. 1977. Variation in number and volume of the mitochondria in yeast according to growth conditions. A study based on serial sectioning and computer graphics reconstitution. Biol. Cellulaire 28: 37-56.

Takano, H., Kawano, S., Suyama, Y. and Kuroiwa, T. 1990. Restriction map of the mitochondrial DNA of the true slime mould, Physarum polycephalum: linear form and long tandem duplication. Curr. Genet. 18: 125-131.

- 1990. Studies on mitochondrial genome of true slime mould, Physarum polycephalum. M. Sc. thesis, University of Tokyo.

Jacob, F., Brenner, S. and Cuzin, F. 1964. On the regulation of DNA replication in bacteria. Cold Spring Hrb. Symp. Quant. Biol. 28: 329-348. 